\section{Clostridium difficile: Infection, diagnosis and treatment with antimicrobial drugs: A review article}

\section{Abstract}

Clostridium difficile infection (CDI) is increasing problem in healthcare, associated with high incidence, mortality, and costs in hospitalized patients. Dramatic increases in the incidence and severity of healthcare-associated $C$. difficile infection have occurred since the last decade, including elderly population, young adults, pregnant females, infants and children. C. difficile infections are mainly linked to the prolonged use of wide-spectrum antibiotics that disrupt the intestinal microbiota equilibrium. Toxigenic strains of $C$. difficile commonly produce two clostridial toxins, toxins $A(\operatorname{Tcd} A)$ and $B(\operatorname{Tcd} B)$, which are responsible for disease symptoms. Few strains of $C$. difficile may also produce another more powerful binary toxin associated with high fatality. The clinical manifestations of infection with toxin-producing strains of $C$. difficile range from symptomless carriage to mild or moderate watery-bloody diarrhea, and few percentage developed fulminant and sometimes fatal pseudomembranous colitis. Complications that have been associated with CDI include dehydration, electrolyte disturbances, toxic megacolon, bowel perforation, hypotension, renal failure, systemic inflammatory response syndrome, sepsis, and death. The most important step in treating CDI is immediately discontinuing use of offending antimicrobial drug. Both metronidazole and vancomycin are equally effective for the treatment of mild CDI, but vancomycin is superior for treating patients with severe $C$. difficile disease. Recently, fidaxomicin proved to be superior to other drugs in treatment of patients who are at high risk for CDI relapse.

Key words: C. difficile, Infection, Diagnosis, Antimicrobial treatment
Asem A. Shehabi, Emman F. Badran, Eman N. Abu-Khader

Departments of Pathology-Microbiology and Pediatrics, Jordan University Hospital, The Jordan University, Amman, Jordan.

Corresponding author:

Dr. Asem A. Shehabi

- ashehabi@go.com.jo ashehabi@ju.edu.jo 
The Genus Clostridium was first described in 1880 , consists of a large number of species with a wide range of biochemical and physiological features. Clostridia are gram positive spore forming bacilli and obligatory anaerobic. Clostridium difficile is present in different rates as part of the indigenous human gut flora. It has also been isolated from diverse natural sources including soils, sand, and the intestinal tracts of animals. The spectrum of disease associated with $C$. difficile ranges from asymptomatic carriage to life-threatening pseudomembranous colitis. Hospitalized patients may frequently develop antibiotic associated colitis or antibiotic associated diarrhea after short or long stay in hospitals [1-2]. In recent years, $C$. difficile infection (CDI) become an increasing heath problem in elderly hospitalized patients and as community-acquired infection, and which can be associated with high incidence, mortality, and healthcare costs $[3,4]$.

\section{Clostridium difficile disease}

The clinical and pathological features of $C$. difficile disease (CDD) indicate that it is difficult to distinguish from other similar intestinal diseases, including ulcerative colitis, chronic inflammatory bowel disease, and Crohn's disease [5]. The clinical manifestations of infection with toxin-producing strains of $C$. difficile range from symptomless carriage, to mild or moderate diarrhea, fulminant and sometimes fatal pseudomembranous colitis. C. difficile diarrhea may be associated with the passage of mucus or occult blood in the stool. Fever, cramping, abdominal discomfort, and a peripheral leukocytosis are also common but found in fewer than half of patients. Extra intestinal manifestations, such as arthritis or bacteremia are very rare [5].

The onset of symptoms after antibiotic treatment in adults has been ranged between 1 day to 6 weeks and longer [6], while the incubation period from antibiotic exposure to develop symptomatic CDI in children is shorter and between two to three days [7]. Almost all patients with $C$. difficile associated disease showed brown or clear watery diarrhea, but less than $50 \%$ have bloody diarrhea $[5,8-9]$. The majority of patients (85\%) with peudomembranous colitis appear to have mucus in their faeces, and their temperature is elevated and exceeds $38^{\circ} \mathrm{C}$. Additionally, leukocytosis is a common clinical feature in the severely ill patient, and complications include dehydration, electrolyte disturbances, toxic megacolon, bowel perforation, hypotension, renal failure, systemic inflammatory response syndrome, sepsis, and death [5]. Complications are more likely to develop among neutropenic children with haematological malignancies or those treated with hematopoietic stem cell transplantation, infants with Hirschsprung's disease and patients with inflammatory bowel disease [7].

Clostridium difficile diseases are mainly linked to the use of wide-spectrum antibiotics that disrupt the intestinal microbiota equilibrium. This allows C. difficile to multiply and colonize the gut [10]. Intestinal colonization is an essential step in the pathogenic process of $C$. difficile and its disease depends on the loss of the commensal microbiota barrier effect following antibiotic treatment as observed in early infancy in infants [10].

According to guidelines from the Infectious Diseases Society of America (IDSA) and the Society for Healthcare Epidemiology of America (SHEA) a case definition of CDI includes the following findings: (a) the presence of diarrhea, defined as passage of 3 or more unformed stools in 24 or fewer consecutive hours1-8, (b) a stool test result positive for the presence of toxigenic $C$. difficile or its toxins, (c) a colonoscopic or histopathologic findings demonstrating pseudomembranous colitis. The same criteria should use to diagnose recurrent CDI. A history of treatment with antimicrobial or antineoplastic agents within the previous 2-month is present in the majority of patients [5].

CDI is defined as hospital-acquired if symptom onset occurred after 48 hours of admission, and 
less than 4 weeks after discharge from a healthcare facility, while CDI was defined as community-acquired if onset of symptoms occurred in the community or within 48 hours of admission to a hospital, pending no clinical symptoms of CDI observed over 12 weeks after the last discharge from a hospital [11]. Watery diarrhea is the most frequent manifestation of CDI in children due to release C. difficile toxins [7].

CDI may be considered if an infant with antibiotic exposure has persistent diarrhea and associated with abdominal findings that persist despite supportive care and absence of typical viral and bacterial pathogens. Additionally, if endoscopy or surgery detects pseudomembranous colitis [12].

\section{Transmission of C. difficile}

The organism is acquired through ingestion of spores usually transmitted from other patients through the hands of health care personnel or the environment. The spores resist the acidity of the stomach and germinate into the vegetative form in the small intestine [9]. The spores of $C$. difficile persist in a dormant state and are difficult to eradicate from the hospital environment and elsewhere [13]. The primary mode of $C$. difficile transmission is person-to-person spread through the fecal-oral route, principally within hospitalized patients. The hands of healthcare workers, are important source for transmission the organism during non-outbreak periods [5]. The major reservoirs for $C$. difficile in the hospital setting are patients with CDAD or asymptomatic carriers of $\mathrm{C}$. difficile who heavily contaminate the hospital environment [14].

\section{Asymptomatic carriage of C. difficile}

Asymptomatic carriage was defined as a positive stool culture or cytotoxin test and the absence of diarrhea during hospitalization and during a 30day period after discharge. Epidemiological Studies have indicated that the prevalence of asymptomatic colonization with $C$. difficile is ranged between $7-26 \%$ among adult inpatients in acute care facilities and is $5-7 \%$ among elderly patients in long-term care facilities $[5,15]$. The risk of colonization increases at a steady rate during hospitalization, suggesting accumulative daily risk of exposure to $C$. difficile spores in the healthcare setting [12]. Newborns and children in their first year of life are known to have some of the highest rates of colonization [5,7]. Infants and children are significantly more likely to carry $C$. difficile asymptomatically in the gastrointestinal (GI) tract than adults. It is estimated that $15-63 \%$ of neonates, $3-33 \%$ of infants and children younger than two years of age, and up to $8.3 \%$ of children older than two years of age are asymptomatic carriers [7]. Infants and young children rarely develop symptoms, possibly because of immature surface intestinal receptors for $C$. difficile, and because they are protected by maternal antibodies acquired transplacentally or in breast milk $[7,16]$. About $51 \%$ of asymptomatic patients carried toxigenic C. difficile, of which $37 \%$ associated with an epidemic strain [17]. Asymptomatic patient is potentially serve as a reservoir for horizontal transmission of epidemic and non-epidemic $C$. difficile strains to other patients, either by contamination of the environment or by contact with hands of medical personnel [18].

\section{Antibiotic-associated diarrhea and pseudomembranous colitis}

Generally mild to moderate diarrhea, sometimes accompanied by lower abdominal cramps is seen with $C$. difficile infection. Symptoms usually begin during or shortly after antibiotic therapy. Occasionally these may be delayed for several weeks [19]. Patients with CDI typically present with watery diarrhea and bloody stools are rare. Patients can be also presented with symptoms of colitis, fever, lower 
abdominal cramps, and fecal leukocytes $[19,20]$. C. difficile toxins can be usually detected in fecal specimens, even though endoscopic and histologic features may be normal in patients with mild disease. The diarrhea resolves with discontinuation of antibiotics with 2-3 days, and the most common clinical manifestation of $C$. difficile infection is colitis without pseudomembrane formation[19]. Sometimes dehydration and a low-grade fever with a systemic polymorphonuclear leukocytosis may occur. A nonspecific diffuse or patchy erythematous colitis without pseudomembrane may be seen under sigmoidoscopy [19, 20].

Pseudomembranous colitis (PMC) is the classic manifestation of full-blown $C$. difficile colitis and is accompanied by similar, but often more severe symptoms than those observed in colitis. The classic pseudomembranes, which are raised yellow plaques ranging from 2-10 $\mathrm{mm}$ in diameter scattered over the colorectal mucosa. White blood cell counts of $20,000 / \mathrm{mm}^{3}$ or greater and hypoalbuminaemia of $3.0 \mathrm{~g} / \mathrm{dl}$ or lower may be observed in severely ill patients [19]. PMC lesions are nearly always limited to the colon. PMC became a commonly recognized complication of antibiotic use in the early 1950s and was primarily encountered by surgeons, who reported rates as high as $14-27 \%$ among postoperative patients. Staphylococcus aureus was the suspected pathogen, and vancomycin given orally became standard treatment for this condition. There are multiple other causes of PMC, including intestinal obstruction, colon cancer, leukemia, severe burns, shock, uremia, heavy metal poisoning, hemolyticuremic syndrome, Crohn disease, shigellosis, neonatal necrotizing enterocolitis, ischemic colitis, and Hirschsprung disease. However, the vast majority of PMC cases seen since 1978 have been attributed to $C$. difficile $[2,5]$. Pseudomembranous colitis can only be diagnosed by direct visualization of pseudomembranes on lower gastrointestinal endoscopy; either by sigmoidoscopy or colonoscopy and by histopathologic examination. However, direct visualization using any of these techniques will detect pseudomembranes in only half of CDI cases that are diagnosed by combined clinical and laboratory tests that include both a culture positive for $C$. difficile and a positive stool cytotoxin test result. Pseudomembranous colitis can be used as a marker of severe $C$. difficile disease [2].

Fulminate colitis occurs in > 5\% Patients with CDI, and patients are severely ill and associated with about $50 \%$ mortality [20]. Patients with fulminant colitis complain of severe lower and diffuse abdominal pain, diarrhea, and distension and some of them may exhibit high fever, chills and marked leukocytosis. Severe protein-losing enteropathy may result in hypoalbuminaemia. A patient with toxic megacolon has a dilated colon with signs and symptoms of severe toxicity that include fever, chills, dehydration and high white blood count [19]. The timing from onset of any CDI symptoms to fulminant colitis varies widely from weeks to just a few hours [20].

Recurrence may result from relapse of the initial infecting strain or due to reinfection with a new strain [21]. Symptomatic recurrence of CDI after successful treatment causes significant morbidity and can prove challenging to treat effectively [22]. Reported recurrence rates vary from 5\% to $50 \%$ and typically are around $20 \%$. Recurrence risk factors included older age, use of provocative antibiotics after CDI diagnosis, concomitant receipt of antacids, hospital-acquired disease, and comorbid conditions, including severe underlying illness or poor quality of life scores. CDI most commonly recurs within a week after finished treatment, but can recur after to 6-8 weeks later. About $50 \%$ of apparent relapses have been identified as new infections with a different strain [23].

\section{Nosocomial infection by C. difficile}

Dramatic increases in the incidence and severity of healthcare-associated C. difficile 
infection have occurred since 2000, particularly in patients over age $65[3,5]$. About 20\% of patients with negative $C$. difficile stool cultures after admission become infected during their hospitalization. Although asymptomatic individuals are capable of shedding spores of $C$. difficile and serve as a reservoir for infection hospitalized patients [24]. New exposure and colonization by $C$. difficile are more likely causing CDI, while patients previously colonized with $\mathrm{C}$. difficile are more likely to remain asymptomatic during their hospitalization [25]. The rate of acquisition of $\mathrm{CDI}$ during hospitalization is proportional to the duration of hospitalization and can be as high as 40\% after 1 month [12]. Different studies showed that $C$. difficile was a major agent of nosocomial diarrhea in adults. The frequency of $C$. difficile or toxins in stool culture prescribed at least 3 days after patients' admission. C. difficile or toxins are recovered from 8-10\% of nosocomial diarrhea [14-15]. One explanation for an increase in both the rate and the severity of $C$. difficile-associated diarrhea could be the emergence of an epidemic strain with increased virulence, antimicrobial resistance or both [26].

\section{Risk factors of CDI}

Several important risk factors for acquiring CDI have been identified and are summarized in Table 1. Antibacterial exposure is the most common risk for the development of CDI. Generally, every antibacterial can be associated with the development of CDI, including ironically metronidazole and vancomycin. The indigenous gut microbiota is normally protect against colonization or infection with C. difficile. Antibacterial can disrupt the competitive balance in the gut microbiota and promote the overgrowth of C. difficile. Several antibacterial classes appear to increase the risk of CDI compared to others, including clindamycin, cephalosporins and fluoroquinolones [1,27]. Recent studies showed that carbapenems place patients at a relatively higher risk for $C D I$, compared with other antibiotics such as first-generation cephalosporins or macrolides [20]. Cumulative antibiotic exposure via dose, use of multiple antibacterial agents, and increased days of antibiotic exposure all contribute to the risk of CDI. Alternatively, limited exposure, such as a single-dose antibacterial exposure for surgical prophylaxis also increases the risk of both $C$. difficile colonization and infection. Some investigators have hypothesized the increased incidence of CDI in young, healthy peripartum women may be a result of exposure to antibacterial prophylaxis associated with Cesarean sections [1]. The second important risk factor is mostly observed among the elderly population. This is most likely due to changes in the intestinal microbiota, an increased use of antibiotic and more frequent hospitalization $[3,28]$. Advanced age has repeatedly been found to be a risk factor for CDI. The risk for CDI has been thought to begin to increase at age 65. It was reported that for each year of age, the risk of health care acquired CDI increases by $2 \%$. The increase in risk could result in part from reduced immune system function with age, particularly of the humoral immune response $[9,20]$. The other risk factors such as gastric acid suppression and host related factors are restricted to small group of patients $[1,29]$.

Table 1. Patient risk factors for initial Clostridium difficile infection [1].

\footnotetext{
Risk for Initial episode

Antibacterial therapy

Advanced age ( $\geq 65$ years)

Gastric acid suppressing agents

Cancer chemotherapy and HIV infection

Enteral feeding and gastrointestinal surgery

Healthcare exposure

Impaired immune response

Underlying chronic comorbidities
} 


\section{Clostridium difficile infection in infants and children}

CDI has been reported in populations previously considered to be at low risk, including young adults, pregnant females, and children. Traditionally, neonates and infants were believed to be asymptomatic carriers of C. difficile; however, recent studies have suggested that $C D I$ is emerging as a cause of diarrhea in infants and children [11]. Moreover, evidence suggests that a large proportion of pediatric CDI cases are community-acquired infections and that many of these infections lack the traditional risk factor of exposure to antimicrobial drugs [29]. The high rate of intestinal colonization of infants with $C$. difficile and the low rates of clinical disease appears to be due to the low capacity of the infant gut to suppress growth of $C$. difficile [3132]. The prevalence of $C$. difficile colonization in neonates ranges from $2 \%$ - 50\% with colonization often occurring within the first week of life. By approximately 2 years of age, colonization rates are similar to those in adults. However, asymptomatically colonize infants represent a potential reservoir for transmission to other family members [12]. It was initially hypothesized that infants were colonized with non-toxigenic strains of $C$. difficile, and this was the reason for the absence of disease. Potential mechanisms for disease resistant in neonates include relatively low numbers of the pathogen in the infant gut, colonization with nontoxigenic, absence of toxin receptors in the immature gut mucosa, and protective components in breast milk [16]. However, multiple studies have now demonstrated the presence of toxin-producing strains in asymptomatic neonates [32]. The American Academy of Pediatrics (AAP) Committee, recommended avoiding routine testing for $C$. difficile in children younger than 1 year of age based on the known high rates of colonization and infrequent disease. They also recommended that testing should be limited in this age group to those with risk factors, as Hirschsprung disease or other severe motility disorders or in an outbreak situation [33]. Recent data have shown that $26 \%$ of pediatric disease that was treated due to CDI occurred in infants younger than one year of age and 5\% occurred in neonates [7]. Treating infants with diarrhea with antibiotics directed against $C$. difficile usually does not alter the course of the diarrhea, even if $C$. difficile is present in the stool [16]. Studies have also demonstrated that mode of delivery by vaginal, cesarean, and instrumental delivery had similar yields of C. difficile in stool of newborn babies. Furthermore, premature rupture of membranes (PROM), sex, and prior administration of antibiotics to the mother or the neonate had no effect on carriage rates. Longer duration of hospital stay of babies appears to increase the prevalence of the carrier state, possibly through increased exposure to C. difficile [16].

\section{Pathogencity and virulence factors of C. difficile}

The indigenous microbiota of the colon provide an important host defense by inhibiting colonization and overgrowth of C. difficile and other potential pathogens [34]. The acid resistance of the organism allows its spores to pass readily through the stomach, enabling germination in the small bowel on exposure to bile acids [35]. C. difficile produces a number of virulence factors that contribute to its virulence. These include adhesion, toxin release, hydrolytic enzyme secretion, flagella, fibronectin-binding protein, antiphagocytic capsule and host factors, and all together contribute to the pathology and feature of infection in the human host $[14,36,37]$.

Toxigenic strains of $C$. difficile commonly produce two large clostridial toxins, toxins $A(\operatorname{Tcd} A)$ and $B$ $(T c d B)$, to which disease symptoms are attributed. They are encoded by genes $t c d A$ and $t c d B$. Together with three additional genes $(t c d C$, $t c d D$, and $t c d E)$, they form the pathogenicity locus (PaLoc), of 19.6 
Table 2. Comparison of Clostridium difficile toxins

\begin{tabular}{|c|c|c|}
\hline Characteristics & Toxin A & Toxin B \\
\hline Molecular weight & $308 \mathrm{kDa}$ & $279 \mathrm{kDa}$ \\
\hline Cytotoxin & + & +++ \\
\hline Intracellular mechanism of cytotoxity & Glycosylated Rho Proteins & Glycosylated Rho Proteins \\
\hline Receptors & Present on enterocyte and other cell & Absent on enterocyte and present on \\
\hline
\end{tabular}

$k b$, which is found only in toxinogenic strains [38]. Both toxins $A$ and $B$ induce mucosal injury and colitis as observed by neutrophil infiltration, which is a prominent feature of CDAD. Toxin $A$ is an enterotoxin that causes haemorrhage and fluid secretion in the intestines of rodents whereas toxin $B$ is a cytotoxin detectable by its cytopathic effects in tissue culture. Toxin A causes extensive damage to the epithelial lining of the intestine and acts as a cytotoxin resulting in disruption of the tight junctions of the intestinal epithelium. Toxin A initially induces cell rounding which results in detachment of the cell from the basement membrane, followed by apoptosis. After toxin $A$ has bound to the receptor initiating the damage, toxin $B$ joins in and gains access to the underlying tissue. The cytotoxic activity of toxin $B$ is similar to that of toxin $A$, but is 1000-fold more potent than the former [19]. The prevalence rates of non-toxigenic $C$. difficile strains in healthy humans are ranged between 10-40\% $[15,17,38]$. These strains do not produce toxins in vivo or in vitro and can colonize the gastrointestinal tract and grow normally in culture media as the toxigenic strains $[15,38]$. Table 2 shows major characteristics of Clostridium difficile toxins.

Some strains of $C$. difficile also produce additive binary toxins CDT; an actin-specific adeninediphosphate (ADP)-ribosyltransferase known as binary toxin CDT, which was first described in 1988 [38]. The binary toxin CDT is unrelated to the wellcharacterized toxins $\mathrm{Tcd} A$ and $\mathrm{TcdB}$. It belongs to the group of clostridial binary toxins, which include the iota toxin of Clostridium perfringens type $E$, the toxin of Clostridium spiroforme, and the C2 toxin of Clostridium botulinum $C$ and D [39]. The binary toxin CDT contains the genes of $c d t A$ and $c d t B$, with an organization and sequences similar to the genes of the iota toxin of $C$. perfringens: the protein sequences of CDTa and CDTb are 81 and 84\% similar, respectively, to the corresponding iota toxin proteins. It was shown that only strains with changes in toxin genes $t c d A$ and $t c d B$ (variant strains) produce binary toxin [40]. Since the majority of strains isolated from symptomatic patients produce only TcdA / TcdB or both, and this indicates that CDT is not required for the virulence of $C$. difficile, but it may serve as an additional virulence factor [39]. Earlier studies found the binary toxin was only present in about $6 \%$ of $C$. difficile clinical isolates [5].. Binary toxin, has been detected recently in $17 \%$ to $23 \%$ of clinical strains, but its role in human disease has not been clearly defined [41].

Increased CDI incidence and severity have been attributed largely to the emergence of a new strain of $C$. difficile, designated by restriction endonuclease analysis type $\mathrm{BI}$, North American pulsed-field gel electrophoresistype1 (NAP1), polymerase chain reaction (PCR) ribotype 027 (BI/NAP1/027) [8,26]. Several characteristics found in BI/NAP1/027 may contribute to its hypervirulence, including polymorphisms in an important toxin production down regulatory gene, tcdC; increased toxin production; 
Table 3. Laboratory diagnosis of Clostridium difficile or its toxin in feces samples

\begin{tabular}{|c|c|c|c|c|}
\hline Test & $\begin{array}{l}\text { Sensitivity } \\
(\%)\end{array}$ & $\begin{array}{c}\text { Specificity } \\
(\%)\end{array}$ & Advantages & Disadvantages \\
\hline $\begin{array}{l}\text { Stool culture \& } \\
\text { biochemical test }\end{array}$ & $90-100$ & $98-100$ & $\begin{array}{c}\text { Allows strain typing } \\
\& \text { antibiotic susceptibility }\end{array}$ & Takes 2-5 days \\
\hline $\begin{array}{l}\text { ElA toxin test for both } \\
\qquad A \& B\end{array}$ & $65-85$ & $95-100$ & $\begin{array}{c}\text { Fast (2-6 h), easy to perform, } \\
\text { high specificity }\end{array}$ & $\begin{array}{l}\text { Less sensitive than other toxin } \\
\text { tests }\end{array}$ \\
\hline Cytotoxin assay & $80-90$ & $99-100$ & Highly sensitive \& specific & $\begin{array}{l}\text { Costly tissue culture, detects only } \\
\text { toxin B within } 24-48 \text { hours }\end{array}$ \\
\hline $\begin{array}{l}\text { Latex agglutination } \\
\text { assay }\end{array}$ & $58-68$ & $80-96$ & $\begin{array}{l}\text { Fast, inexpensive, easy to } \\
\text { perform }\end{array}$ & $\begin{array}{l}\text { Low sensitivity to detect toxin in } \\
\text { stool }\end{array}$ \\
\hline $\begin{array}{l}\text { PCR for detection } \\
\text { toxin genes }\end{array}$ & $92-97$ & 100 & $\begin{array}{l}\text { Excellent sensitivity and } \\
\text { specificity compared to all tests }\end{array}$ & Done mostly in Research labs \\
\hline
\end{tabular}

presence of the gene encoding binary toxin (ctdA and $\operatorname{ctd} B$ ); high-level fluoroquinolone resistance and polymorphisms in $t c d B$ that could result in improved toxin binding [8]. This toxigenotype (NAP1/027) can produce 16 times more toxin $\mathrm{A}$ and 23 times more toxin B than control strains [42]. The NAP1 strain has entered the pediatric population at lower rates (10-19\%) of toxigenic C. difficile isolates than reported for adults (>50\%) [18,43].

\section{Laboratory diagnosis of C. difficile infection}

Rapid and accurate diagnosis of CDI is essential for improving outcomes of patients and the overall management of its nosocomial infection [44]. The diagnosis of CDI should be based on a combination of clinical and laboratory findings [4].

The medium cycloserine-cefoxitin fructose agar (CCFA) is the most used selective culture for recovery of $C$. difficile [5]. The second commonly used $C$. difficile moxalactam-norfloxacin agar plates (CDMN) is also highly selective for its isolation[45]. The selection of spores from feces samples by alcohol shock greatly diminishes almost all competing fecal flora and enhances both the isolation and easier recognition of $C$. difficile. The addition of bile salts, such as sodium cholate or taurocholate, to the culture medium is believed to enhance the recovery of spores by inducing the germination of spores from environmental samples or faeces after alcohol shock [46]. Optimal results require that culture plates be reduced in an anaerobic environment prior to use. The colonies are flat, yellow and have a typical odor and fluoresce with a Wood's lamp. Additionally, Gram stain shows typical morphology of gram-positive or gram-variable bacilli [5]. There are several biochemical tests, cytotoxin assay and immunological test available to confirm the identity of $C$. difficile or to detect toxin. Each test has unique characteristics as presented in Table $\mathbf{3}$.

Detection of $\mathbf{C}$. difficile Toxins: Toxin B (cytotoxin) which is produced by toxigenic strains of $C$. difficile can be detected by different cell lines such as Vero, HEP-2 or MRC-5 monkey kidney and HeLa cell lines [5]. The first enzyme immunoassay (EIA) for detecting C. difficile toxin in fecal samples was developed and evaluated by Yolken et al., in 1981 [47]. Commercial EIA tests have been introduced that either detect toxin A only or detect both toxins A and B. It has been the most widely used diagnostic test for CDI [8].

Detection of $\boldsymbol{C}$. difficile Toxin by Molecular Methods: Several nucleic acid amplification tests 
(NAATs) are FDA approved for C. difficile testing. These assays detect conserved regions of toxin $A$ or toxin B genes on the PaLoc of C. difficile [48]. NAAT utilizing either polymerase chain reaction (PCR) with excellent sensitivity (92\%-97\%) and specificity (100\%) or loop-mediated isothermal amplification (LAMP), which appear to have similar performances is increasingly being used for diagnosis of $C$. difficile infection and healthy carriers who have diarrhea from unrelated causes [49]. (Gould et al., 2013). Although more expensive than traditional assays, these tests have potential for rapid and accurate diagnosis [44].

For epidemiological purpose, there are various methods currently adapted globally to type C. difficile isolates, including pulsed-field gel electrophoresis (PFGE), PCR ribotyping, toxinotyping based on restriction fragment length polymorphism (RFLP), restriction endonuclease analysis (REA), multilocus variablenumber tandem-repeat analysis (MLVA), and multilocus sequence typing (MLST). [50].

\section{Treatment of $C$. difficile clinical cases with antibiotics}

The most important step in treating $C D I$ is immediately discontinuing use of the offending antimicrobial drug; and the appropriate antibiotic should be treated the patient. Additionally, providing appropriate supportive care with rehydration and electrolyte replacement as needed, and avoiding the use of antiperistaltic agents which may contribute to the development of toxic megacolon $[12,51]$. Once the offending antibiotic is discontinued, spontaneous resolution of CDAD symptoms will be observed in most patients within 2-4 days $[5,51]$. Metronidazole (Flagyl) and oral vancomycin have been the main antimicrobial agents used in the treatment of CDI. The traditional therapy for patients with moderate to severe CDI is either oral metronidazole (400 mg 8 hourly) or oral vancomycin (125mg 6 hourly) for 10-14 days. Relapse rates of $C$. difficile have been noted as high as 20\% with metronidazole and $30 \%$ with vancomycin. A study has compared oral metronidazole $250 \mathrm{mg}$ four times daily to oral vancomycin 250 mg four times daily, and the results suggested that oral vancomycin was superior for patients severed by severe $C$. difficileassociated disease, although the study results did not reach statistical significance [12]. Metronidazole is currently preferred in guidelines from the Centers for Disease Control and Prevention (CDC), Health Protection Agency in UK (HPA) (HPA, 2009), the Infectious Diseases Society of America (IDSA), and the Society for Healthcare Epidemiology of America (SHEA), on the basis of low cost and the concern that oral vancomycin promotes colonization with vancomycin-resistant enterococci (VRE) $[5,20]$. When administered orally, metronidazole is absorbed rapidly and almost completely, with only $6-15 \%$ of the drug excreted in stool. In contrast, vancomycin is poorly absorbed, and fecal concentrations after oral administration reach high levels [12]. The drug of choice for seriously ill patients is oral vancomycin (only approved by the US Food and Drug Administration (FDA) for CDI), because it has no side effect and is not absorbed by the intestine [19]. Metronidazole can be associated with severe allergic reactions as well as central nervous system toxicity. The neurotoxicity associated with metronidazole is related to cumulative exposure, and thus occurs at a higher rate following prolonged therapy, as is often used in cases where repeat $C$. difficile testing remains positive [32]. Treatment does not eradicate $C$. difficile or the toxin from the stool. Asymptomatic patients, should not be treated again if the stool test was still positive [7]. For first and second episodes of diarrhea, metronidazole is still recommended, although oral vancomycin should be considered when the patient is experiencing sepsis associated with C. difficile infection [20]. Newer antimicrobial agents with activity against $C$. difficile have been developed and studied in adults, 
including fidaxomicin (FDA approved for treatment of CDI in adults in 2011), nitazoxanide, and rifaxamin but experience with their use in children is limited to date [32].

\section{Antimicrobial resistance in C. difficile}

Drug resistance of C. difficile towards metronidazole is still not common, while total resistance to vancomycin is not documented over the world [52]. In a recent study from Spain, a resistance rate for metronidazole (minimum inhibitory concentration $32 \mathrm{mg} / \mathrm{L}$ ) was noted at $6.3 \%$. It is expected that prolonged exposure to metronidazole can be linked to resistance. An earlier study from Spain in 2002 noted that 3\% of 415 C. difficile isolates showed intermediate susceptibilities (MIC $4 \mathrm{mcg} / \mathrm{mL}-16 \mathrm{mcg} / \mathrm{mL}$ ) to vancomycin [53]. Vancomycin contributes to higher selection pressure than metronidazole in developing resistance in enterococci. With the concerns of high rates of relapse, drug resistance and treatment failures, other agents are being investigated as alternative treatment strategies for CDI[52]. Furthermore, an increased rate of $C$. difficile strains show resistance to rifamycins, used for the treatment of relapsing CDI has been reported [20,52].

Several mechanisms of resistance have been identified in C. difficile, including acquisition of genetic elements and alterations of the antibiotic target sites. The $C$. difficile genome contains mobile genetic elements, many of them involved in antibiotic resistance. Transfer of genetic elements among $C$. difficile strains or between C. difficile and other bacterial species can occur through different mechanisms that facilitate their spread [54]. Antibiotic resistance plays an important role in the emergence of new $C$. difficile types. While clindamycin resistance was observed in endemic types, new epidemic types are associated with resistance to fluoroquinolones
[55]. Furthermore, resistance to multiple antibiotics is a common feature observed in recently emergent epidemic isolates.

A recent Pan-European Longitudinal Surveillance has reported there was no evidence of resistance to new used fidaxomicin, and reduced susceptibility to metronidazole and vancomycin was also scarce. Rifampicin, moxifloxacin, and clindamycin resistance accounted to $13 \%, 40 \%$, and $50 \%$ of total isolates, respectively and were associated with multiple ribotypes [56]. The rapid identification of new phenotypic and genotypic traits, the implementation of effective antimicrobial stewardship and infection control programs, use of alternative drugs are all important factors to prevent and control the spread of resistance and to ensure a successful therapy for CDI.

\section{Biotherapy of C. difficile clinical cases}

Probiotics such as lactobacillus species and Saccharomyces boulardii, have shown efficacy in reducing the incidence of simple antibioticassociated diarrhea, but their efficacy in preventing C. difficile infection is not consistent, however, reestablishment of a healthy intestinal flora is the aim of probiotics $[20,57]$. There are limited data to support this treatment approach and there is a potential risk of bloodstream infection. Until recently, no single study had shown clearly probiotics to be effective in the prevention of CDI [5].

\section{Clostridiun difficile infection in Arab countries}

There are few epidemiologic data published on C. difficile in Arab countries over the last 15 years according the search in 2 most medical famous indexing web sites (Pub Med \& Scopus). In Jordan, 
Shehabi et al. [9], performed a study at the Jordan University Hospital in 2000 and found a prevalence rate of $9.7 \%$ C. difficile isolates or its toxin in patient stools of all ages with diarrhea by using culture and enzyme immunoassay for the detection of $C$. difficile toxin A. A study done in Kuwait by Rotimi et al. in 2002 [58], reported that the prevalence of hospital acquired C. difficile infection/colonization was less than 10\%. A second study conducted in Kuwait focused on the PCR ribotyping of environmental and ICU clinical strains. A total of 32 different ribotypes were detected among the clinical isolates, and the predominant toxigenic ribotypes detected were types 097 and 078, which are different from the findings obtained in North America and Europe, which exhibited a dominance of the 027 ribotype [59].

Nasereddin et al. [15], reported an increase in CDI rates observed at the Jordan University Hospital after abot 8 years of the first study done in the same hospital. The study showed a prevalence of toxigenic C. difficile isolates was 13.7\% among adult hospitalized patients, and $73 \%$ of the $C$. difficile isolates carried tcdA and/or tcdB toxin genes as demonstrated by PCR and in association with diarrhea, and all C. difficile isolates were negative for binary toxin production. A recent multicenter study held in Jordan in 2015 by Wadi et al. [3], detected a prevalence rate of $92.4 \%$ among hospitalized old patients with $C$. difficile toxin-positive diarrhea-stools using a rapid test, which detects fecal $C$. difficile toxins $A$ and $B$. The adults and older age groups accounted for the majority of all positive cases. In Saudi Arabia, a study reported a prevalence rate of $4.6 \%$ of C. difficile among Saudi patients. Stool analysis for $C$. difficile toxins $A$ and $B$ was carried out by an enzyme-linked immunosorbent assay [60]. In Qatar, Al-Thani et al. [61], recently reported a prevalence rate of CDI (7.9\%) amongst their 1,532 hospitalized patients, and their study used glutamate dehydrogenase (GDH) lateral flow assay and toxins $A$ and B Enzyme Immunoassay (EIA) fordetection of
C. difficile. A recent Lebanese study demonstrated that 30 out 88 (65.2\%) of stool samples examined of patients admitted to different hospital units at the American University Medical Center in Beirut, were positive by culture for $C$. difficile or its toxins (tcdA/ $\operatorname{tcd} B$ ) or both genes. Their results of toxinotyping showed that 2 isolates belonged to toxinotype 0, 4 to toxinotype $\mathrm{XI}, 2$ to toxinotype $\mathrm{XII}, 1$ to toxinotype $\mathrm{XVI}, 1(\mathrm{~A}+\mathrm{B}-)$ and twenty $(\mathrm{A}-\mathrm{B}+)$ designated as toxinotype 0-like [62]. The frequency, demography, clinical features and outcome of nosocomial CDAD in children admitted to Assiut University Children's Hospital, Egypt, was investigated by a descriptive cross-sectional study. Out of 72 examined children, $17(23 \cdot 6 \%)$ were diagnosed with CDAD using culture for $C$. difficile and direct toxin detection from stool samples by enzyme immuno-assay. Those aged $\leq 12$ months were the most commonly affected (eight, $47 \%)$. The main cause of admission was respiratory disorders (47\% of cases) [63].

Lastly, a new not published yet study from Jordan (personal communication, 2016), indicated that the overall C. difficile colonization rate among Jordanian infants aged less than one year was (37/287; $12.9 \%)$. Both toxigenic (54\%) and non-toxigenic C. difficile strains (46\%) were detected. Additionally only one isolate $(2.7 \%)$ was positive for binary toxin. All isolates of $C$. difficile were susceptible to vancomycin and metronidazole, while high resistance rate to ciprofloxacin and low resistance rate to erythromycin were detected among the isolates. The results of fluoroquinolone resistance determining-genes indicated that (40.5\%) of the isolates carried both gyrA and gyrB.

The presence of toxigenic $C$. difficile in stools of infants without diarrhea, suggests that they were asymptomatic carriers of toxigenic strains; thus infants may be a potential reservoir to spread infection with these trains to other close contact.

The authors acknowledge that they have no conflict of interest. 


\section{References}

1. DePestel DD, Aronoff DM. Epidemiology of Clostridium difficile Infection. J Pharm Pract 2013; 26(5): 464-475.

2. Bartlett JG. Historical Perspectives on Studies of Clostridium difficile and C. difficile Infection. Clin Infect Dis 2008; 46, 4-11.

3. Wadi J, Ayesh, AS, Abu Shanab L, Harara B, Petro H, et al. Prevalence of Clostridium difficile infections among hospitalized patients in Amman, Jordan: A Multi-Center Study. The IAJAA 2015; 5 No. 1:1 doi: 10.3823/763.

4. Gupta A, Khanna S. Community-acquired Clostridium difficile infection: an increasing public health threat. Infect Drug Resist $2014 ; 7,63-72$.

5. Cohen SH, Gerding DG, Johnson S, Kelly CP, Loo VG, et al. Clinical Practice Guidelines for Clostridium difficile Infection in Adults: 2010 Update by the Society for Healthcare Epidemiology of America (SHEA) and the Infectious Diseases Society of America (IDSA). Infect Cont Hosp Epidemiol 2010; 31,431-455.

6. Poutanen SM, Simor AE. Clostridium difficile-associated diarrhea in adults. Cana Med Associat J 2004; 171 (1): 51-58.

7. Allen UD, Canadian Pediatric Society, Infectious Diseases and Immunization Committee. Clostridium difficile in pediatric populations. Paediatr Child Health, 2014;19(1): 43-48.

8. Lessa FC, Gould CV, McDonald LC. Current Status of Clostridium difficile Infection Epidemiology. Clin Infect Dis 2012; 55(S2):S65-70.

9. Shehabi AA, Abu-Ragheb, HA, Allaham NA. Prevalence of Clostridium difficile- associated diarrhea among hospitalized Jordanian patients. Eastern Medit Health J 2001 7:750-755.

10. Rousseau C, Levenez, F, Fouqueray L, Dore J, Collignon A, et al. Clostridium difficile Colonization in Early Infancy Is Accompanied by Changes in Intestinal Microbiota Composition. J Clin Microbiol 2011; 49, 858-865.

11. Khanna S, Baddour LM, Huskins WC, Kammer PP, Faubion WA, et al. The Epidemiology of Clostridium difficile Infection in Children: A Population-Based Study. Clin Infect Dis 2013; 56(10):1401-6.

12. Tamma PD, Sandora TJ. Clostridium difficile Infection in Children: Current State and Unanswered Questions. J Pediatric Infect Dis Soc 2012; 1(3), 230-43.

13. Pawar D, Bhandari P, Allenby K. Clostridium difficile-Associated Diarrhea: A Review. Indian Med Gaz 2011; 145 (12):481-494. Deneve C, Janoir C, Poilane I, Fantinato C, Collignon A. New trends in Clostridium difficile virulence and pathogenesis. Int J Antimicrob Agents 2009;33 Suppl 1:S24-8.

14. Barbut F, Petit JC. Epidemiology of Clostridium difficileassociated infections. Clin Microbiol Infect 2001; 7, 405-410.
15. Nasereddin LM, Al-Bakri FG, Shehabi AA. Clostridium difficile infections among Jordanian adult hospitalized patients. Amer J Infect Control 2009; 10: 864-866.

16. Jangi S, Lamont JT. Asymptomatic colonization by Clostridium difficile in infants: implications for disease in later life. Journal of Pediatric Gastroenterology and Nutrition 2010; 51(1):2-7.

17. Hung YP, Lee, JC, Lin HJ, Liu HC, et al. Clinical impact of Clostridium difficile Colonization. J Microbiol Immunol Infect 2015; 48, 241-248.

18. Riggs MM, Sethi AK, Zabarsky TF, Eckstein EC, Jump RP, et al. Asymptomatic carriers are a potential source for transmission of epidemic and nonepidemic Clostridium difficile strains among long-term care facility residents. Clinical Infectious Diseases 2007;45 (8): 992-998.

19. Vaishnavi C. Clinical spectrum \& pathogenesis of Clostridium difficile associated diseases. IJMR 2010; 31, 487-499.

20. McCollum DL, Rodriguez JM. Detection, Treatment, and Prevention of Clostridium difficile Infection. Clin Gastroenterol Hepatol 2012;10, 581-592.

21. Fekety R, McFarland LV, Surawicz CM, Greenberg RN, Elmer GW. et al. Recurrent Clostridium difficile Diarrhea: Characteristics of and Risk Factors for Patients Enrolled in a Prospective, Randomized, Double-Blinded Trial. Clin Infect Dis 1997; 24:32433.

22. Kelly CP, LaMont, JT. Clostridium difficile-more difficult than ever. N Engl J Med 2008; 359:1932-40.

23. Eyre DW, Walker AS, WyllieD, Dingle KE, Griffiths D. et al. Predictors of First Recurrence of Clostridium difficile Infection: Implications for Initial Management. Clin Infect Dis 2012; 55(S2):S77-87.

24. Curry SR, Muto CA, Schlackman JL, Pasculle AW, Shutt, KA, et al. Use of Multilocus Variable Number of Tandem Repeats Analysis Genotyping to Determine the Role of Asymptomatic Carriers in Clostridium difficile Transmission. Clinical Infectious Diseases 2013; 57(8):1094-102.

25. Blossom DB, McDonald LC. The Challenges Posed by Reemerging Clostridium difficile Infection. Clin Infect Dis 2007; 45:222-7.

26. McDonald LC, Killgore GE, Thompson A, Owens RC, Kazakova $S V$, et al. An epidemic, toxin gene-variant strain of Clostridium difficile. N Engl J Med 2005; 353(23):2433-41.

27. Cheng AC, Ferguson JK, Richards MJ, Jennifer MR, Gwendolyn $L G$, et al. Australasian Society for Infectious Diseases guidelines for the diagnosis and treatment of Clostridium difficile infection. M J A 2011; 194:353-358. Drekonja DM, Amundson WH, Decarolis DD et al. Antimicrobial use and risk for recurrent Clostridium difficile infection. Am J Med 2011;124:1081 e1-7.

28. Rea MC, O'Sullivan O, Shanahan F, O'Toole PW, Stanton, $C$, et al. Clostridium difficile Carriage in Elderly Subjects and 
Associated Changes in the Intestinal Microbiota. J Clin Microbiol 2012; 867-875.

29. HungYP, Lin HJ, Wu TC, Liu HC, Lee JC, et al. Risk Factors of Fecal Toxigenic or Non-Toxigenic Clostridium difficile Colonization: Impact of Toll-Like Receptor Polymorphisms and Prior Antibiotic Exposure. PLoS ONE 2013; 8(7): e69577.

30. Zilberberg MD, Tillotson GS, McDonald LC. Clostridium difficile Infections among Hospitalized Children, United States, 1997-2006. Emerg Infect Dis 2010; 16(4), 604-609.

31. Adlerberth I, Huang $H$, Lindberg $E$, Aberg N, Hesselmar B, et al. Toxin-Producing Clostridium difficile Strains as Long-Term Gut Colonizers in Healthy Infants. J Clin Microbiol 2014; 52, 173 179.

32. Nicholson MR, Thomsen IP, and Edwards KM. Controversies Surrounding Clostridium difficile Infection in Infants and Young Children. Children 2014; 1, 40-47.

33. Borali E, Ortisi G, Moretti C, Stacul EF, Lipreri R, etal. Communityacquired Clostridium difficile infection in children. Digest Liver Dis 2015; 47(10):842-6.

34. Owens RC, Curtis J, Donskey J, Gaynes RP, Loo VG, et al. Antimicrobial-Associated Risk Factors for Clostridium difficile Infection. Clin Infect Dis 2008;46:S19-31.

35. Poutanen SM, Simor AE. Clostridium difficile-associated diarrhea in adults. CMAJ 2004;171 (1): 51-58.

36. Sunshine RH, McDonald LC. Clostridium difficile-associated disease: New challenges from an established pathogen. CCJM 2006; 73, 187-197.

37. Borriello SP. Pathogenesis of Clostridium difficile infection. J Antimicrob Chemother 1998; 41, 13-19.

38. Geric B, Rupnik M, Gerding DN, Grabnar M, Johnson S. Distribution of Clostridium difficile variant toxinotypes and strains with binary toxin genes among clinical isolates in an American hospital. J Med Microbiol 2003; 53, 887-894.

39. Goncalves C, Decree D, Barbut F, Burghoffer B, Petit JC. Prevalence and Characterization of a Binary Toxin (ActinSpecific ADP-Ribosyltransferase) from Clostridium difficile. J Clin Microbiol 2004; 42, 1933-1939.

40. Stubbs S, Rupnik M, Gibert M, Brazier J, Duerden B, et al. Production of actin-specific ADP-ribosyltransferase (binary toxin) by strains of Clostridium difficile. FEMS Microbiology Letters 2000; 186, 307-312.

41. Eckert C, Emirian, A, Monnier A, Cathala L, Montclos $H$, et al. Prevalence and pathogenicity of binary toxin-positive Clostridium difficile strains that do not produce toxin A and B. New Microbes New Infec 2015; 3, 12-17.

42.Warny M, Pepin J, Fang A, Killgore G, Thompson A. et al. Toxin production by an emerging strain of Clostridium difficile associated with outbreaks of severe disease in North America and Europe. Lancet 2005; 366:1079-84.

43. Schutze GE, Willoughby RE. Clostridium difficile infection in infants and children, Committee on Infectious Diseases. Pediatrics 2013; 2013;131:196.

44. O'Horo, JC, Jones A, M,Harper C, Safdar, N. Molecular Techniques for Diagnosis of Clostridium difficile Infection: Systematic Review and Meta-analysis. Mayo Clin Proc 2012; 87(7): 643-651.

45. Aspinall TS, ND. New selective medium for isolating Clostridium difficile from faeces. J Clin Pathol 1992; 45(9): 812-814.

46. Brazier JS. The diagnosis of Clostridium difficile-associated disease. J of Antimicrob Chemoth 1998;41, 29-40.

47. Yolken RH, Whitcomb LS, Marien G. Enzyme immunoassay for the detection of Clostridium difficile antigen. J Infect Dis 1981;144:378.

48. Carroll KC, Loeffelholz, M. Conventional versus Molecular Methods for the Detection of Clostridium difficile. J Clin Microbiol 2011;49(9): S49-S52.

49. Gould CV, Edwards, JR, Cohen J, Bamberg WM, Clark LA, etal. Effect of Nucleic Acid Amplification Testing on PopulationBased Incidence Rates of Clostridium difficile Infection. Clin Infect Dis 2013; 57(9):1304-7.

50. Van Belkum A, Tassios PT, Dijkshoorn L, Haeggman S, Cookson B, et al. (2007), European Society of Clinical Microbiology and Infectious Disease (ESCMID) Study Group on Epidemiological markers (ESGEM). Guidelines for the validation and application of typing methods for use in bacterial epidemiology. Clin Microbiol Infect Dis 2007; 13, 1-46.

51. Khan FY, Elzouki A. Clostridium difficile infection: a review of the literature. Asian Pacif J Trop Med, 7(1): S6-S13.

52. Patrizia Spigaglia. Recent advances in the understanding of antibiotic resistance in Clostridium difficile infection. Ther Adv Infect Dis 2015; 2049936115622891

53. Weber I, Riera E, Déniz C, Pe'rez J, Oliver A, et al. Molecular epidemiology and resistance profiles of Clostridium difficile in a tertiary care hospital in Spain. Int J Med Microbiol 2013; 303: 128-133.

54. Jasni A, Mullany, $P$, Hussain $H$, Roberts A. Demonstration of conjugative transposon (Tn5397)-mediated horizontal gene transfer between Clostridium difficile and Enterococcus faecalis. Antimicrob Agents Chemother 2010; 4: 4924-4926

55. Spigaglia P, Barbanti F, Dionisi AM, Mastrantonio P. Clostridium difficile isolates resistant to fluoroquinolones in Italy: emergence of PCR ribotype 018. J Clin Microbiol 2010;48(8):2892-6.

56. Freeman J, Vernon J, Morris K, Nicholson S, Todhunter S, et al. Pan-European Longitudinal Surveillance of Antibiotic Resistance 
among Prevalent Clostridium difficile Ribotypes' Study Group. Clin Microbiol Infect. 2015; 21(3): 248.e9-248.

57. Kelly, CP, LaMont, JT. (2008), Clostridium difficile-more difficult than ever. N Engl J Med 2008;359:1932-40.

58. RotimiVO, Mokaddas EM, Jamal WY, Verghese TL, El-Din K, et al. Hospital-acquired Clostridium difficile infection amongst ICU and bum patients in Kuwait. Med Principles Pract 2002; 11: 2328.

59. Rotimi, VO. Jamal WY, Mokaddas EM, Brazier JS, Johny M, et al. Prevalent PCR ribotypes of clinical and environmental strains of Clostridium difficile isolated from intensive-therapy unit patients in Kuwait. J Med Microbiol 2003; 52: 705-709.

60. Al-Tawfiq JA, Abed MS. Clostridium difficile-associated disease among patients in Dhahran, Saudi Arabia, Travel Med Infect Dis 2010; 8(6):373-6.

61. Al-Thani AA, Hamdi WS, Al-Ansari NA, Doiphode SH. Polymerase chain reaction ribotyping of Clostridium difficile isolates in Qatar: a hospital-based study. BMC Infectious Diseases 2014;14:502.

62. Moukhaiber R, Araj GF, Kissoyan KA, Cheaito KA, Matar GM. Prevalence of Clostridium difficile toxinotypes in infected patients at a tertiary care center in Lebanon. J Infect in Dev Ctries 2015; 9(7):732-735.

63. Abu FaddanNH, AlySA, Abou Faddan HH. Nosocomial Clostridium difficile-associated diarrhoea in Assiut University Children's Hospital,Egypt. Paediatr Int Child Health 2015; Dec 29. [Epub ahead of print]

\section{Comment on this article:}
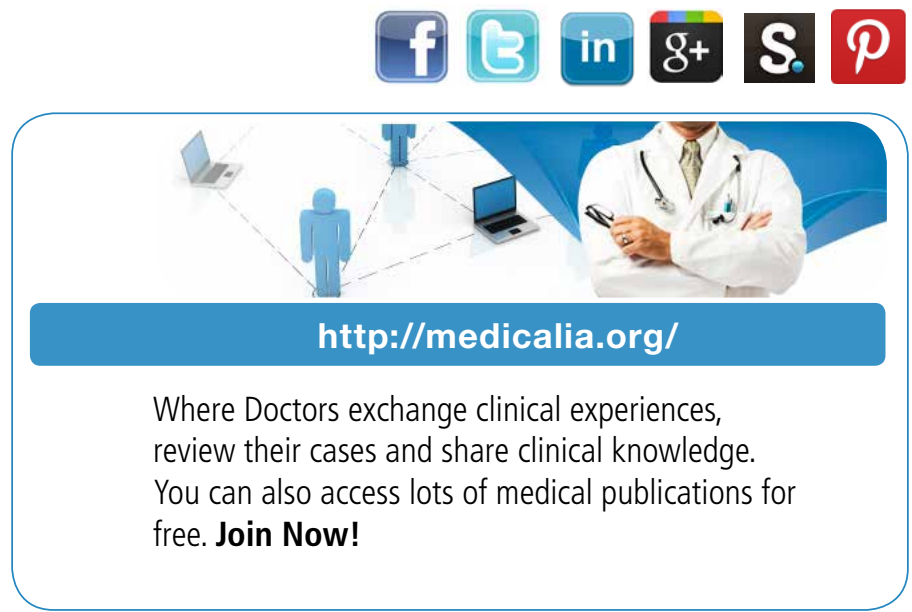

\section{Publish with iMedPub}

http://www.imed.pub

The Journal is an open access peer-reviewed journal that publishes scientific papers about all aspects of antimicrobials. The journal will publish original research articles, reviews, brief reports and case reports dealing with basic and clinical antibacterial agents, antiviral, antiprotozoals, antituberculuous, antifungal and antihelminthes agents.

All manuscripts must be prepared in English, and are subject to a rigorous and fair peer-review process. Accepted papers will immediately appear online.

The journal aims to advance the knowledge, attitude and the research of chemotherapy in the Arabic world in cooperation with international, national scientific and public societies as well as research centers with similar aims and objectives. 